\title{
NSW HEALTH ABORIGINAL HEALTH IMPACT STATEMENT
}

\section{Liz Wheeler}

Centre for Aboriginal Health

NSW Department of Health

Health outcomes for Aboriginal people remain significantly poorer than those for the general population. For example, the average life expectancy is 56 years for Aboriginal men and 64 years for Aboriginal women. This is approximately 20 years less than the life expectancy for the general population. ${ }^{1}$

There has been little improvement in recent decades in the health of Aboriginal and Torres Strait Islander peoples, in contrast to the progress that has been made in improving the health of indigenous peoples in countries such as New Zealand, the United States and Canada. It is widely recognised that public health strategies can play a key role in improving the health of indigenous peoples. ${ }^{2}$

Within the health system, an important mechanism for improving Aboriginal and Torres Strait Islander health is the availability of comprehensive primary health care services. Increasing the level of resources to reflect the higher level of need of Aboriginal and Torres Strait Islander peoples; improving access to both mainstream and Aboriginal and Torres Strait Islander specific health and health-related programs which reflect the higher level of need; joint planning processes and improved data collection and evaluation $^{3}$ are a priority for all governments.

However, health strategies, policies and programs often reflect the values and culture of the decision-makers and other senior managers who design them. Consequently, principles and guidelines are needed to assist in the development of policies and programs that reflect the values and needs of the Aboriginal community. ${ }^{3}$

To meet this need, the Centre for Aboriginal Health at the NSW Department of Health developed the NSW Health Aboriginal Health Impact Statement (the Statement). ${ }^{4}$ This was done in partnership with the Aboriginal Health and Medical Research Council of NSW, a peak body representing the Aboriginal community controlled health sector in NSW, Area Directors of Aboriginal Health from the area health services in NSW, and other stakeholders. The Statement is based on the core Aboriginal health principles, which are designed to guide all relevant health policy initiatives: a whole-of-life view of health, selfdetermination, partnership, cultural understanding and recognition of loss and trauma..$^{5}$ The Statement drew upon the National Public Health Partnership's Guidelines for the development, implementation and evaluation of national public health strategies in relation to Aboriginal and Torres Strait Islander peoples ${ }^{6}$, which were released in February 2002,
The purpose of the Statement is to ensure that the needs and interests of Aboriginal peoples in NSW are integrated into the policy, program and service development process. It is also used to identify any effects that an initiative is likely to have on Aboriginal health outcomes and to ascertain what additional resources may be needed to address the effects.

Effective Aboriginal and Torres Strait Islander representation is central to the success of Aboriginal specific strategies. ${ }^{2}$ The Statement therefore contains a series of questions to help determine whether appropriate Aboriginal consultation and negotiation processes have taken place.

Completing a Statement is mandatory for all NSW Health staff and consultants who have been engaged by NSW Health in the development, implementation or evaluation of policies, services or programs that may have direct or indirect effects on Aboriginal health.

The approach taken by the Aboriginal Health Impact Statement is also consistent with the NSW Government's 10-year Aboriginal Affairs Plan, Two Ways Together ${ }^{6}$, which requires all government agencies to develop policies, procedures and protocols recognising Aboriginal culture, needs and aspirations.

A trial implementation phase for the NSW Health Aboriginal Health Impact Statement has been completed and the Statement has been redrafted. Full statewide implementation is planned by June 2006.

\section{REFERENCES}

1. Stewart G. The health of the people of New South Wales: Report of the Chief Medical Officer, Sydney, NSW Department of Health, 2002, 83.

2. The National Public Health Partnership. Guidelines for the Development, Implementation and Evaluation of National Public Health Strategies in relation to Aboriginal and Torres Strait Islander Peoples. Report for the National Public Health Partnership. Melbourne, Australia: National Public Health Partnership; 2002. Available at: www.dhs.vic.gov. au/nphp/publications/strategies/atsi_guidelines.pdf 3 . Commonwealth of Australia. National strategic framework for Aboriginal and Torres Strait Islander health 2003-2013Framework for action by governments, 2004.

3. NSW Health. NSW Health Aboriginal health impact statement and guidelines, Sydney; NSW Department of Health, 2003.

4. NSW Health. Ensuring progress in Aboriginal health: A policy for the NSW health system. NSW Health and the Aboriginal and Medical Research Council of NSW; 2002.

5. NSW Department of Aboriginal Affairs. Two ways together: New South Wales Aboriginal Affairs Plan 2003-2012. Sydney: NSW Department of Aboriginal Affairs, 2003. Available from: www.daa.nsw.gov.au/data/files//TWTbrochure.pdf. 